\title{
COVID-19 Electronic Registry Systems in Iran: A Review
}

\author{
Razieh Sadat Mousavi-Roknabadi1,2*(1) , Hosain Safaei-Firouzabadi ${ }^{(\infty)}$, Reyhaneh Sadat Mousavi- \\ Roknabadi $^{4}$, Mehrdad Sharifi ${ }^{1,2}$, Robab Sadegh ${ }^{1,2}{ }^{\circledR}$, Mojtaba Mokdad ${ }^{5}$ \\ ${ }^{1}$ Emergency Medicine Department, School of Medicine, Shiraz University of Medical Sciences, Shiraz, Iran \\ ${ }^{2}$ Emergency Medicine Research Center, Shiraz University of Medical Sciences, Shiraz, Iran \\ ${ }^{3}$ Department of Civil Engineering, Yazd Branch, Islamic Azad University, Yazd, Iran \\ ${ }^{4}$ School of Medicine, Yazd Branch, Islamic Azad University, Yazd, Iran \\ ${ }^{5}$ Gomel State Medical University, Gomel, Belarus
}

Corresponding Author: Razieh Sadat Mousavi-Roknabadi, MD, Community Medicine Specialist, Emergency Medicine Department, School of Medicine, Shiraz University of Medical Sciences, Shiraz, Iran. Tel: +98-

9131563018, Email: mousavi_razieh@sums.ac.ir

Received Aprill 11, 2021; Accepted June 22, 2021; Online Published August 11, 2021

\begin{abstract}
Introduction: Since the beginning of the COVID-19 epidemic in Iran, several electronic registration systems have been created to record the data of infected patients. This narrative review aimed to investigate the articles that described the COVID-19 electronic registry systems designed and implemented in Iran.

Methods: In this review, four electronic databases [Medline (accessed from PubMed), Scopus, Science Direct, and Web of Science] were searched till June 22, 2020, using specific MeSH terms and related keywords in English language. Considering the titles and abstracts, unrelated studies were excluded. The full texts of the remained studies were evaluated by authors, independently. Then, their findings were assessed and reported.

Results: Finally, four articles were enrolled, introducing four COVID-19 registries. These registries were designed and launched by Isfahan, Shiraz, Tehran, and Ilam Universities of Medical Sciences. They were different in design, the used algorithms for patients' management, recorded data, and methods of quality assurance.

Conclusion: Considering the differences between various registry systems designed for COVID-19 in Iran, it is recommended to develop a single web-based registry system by the Iran Ministry of Health and Medical Education to register and follow up the patients with COVID-19.

Keywords: Coronavirus, COVID-19, Registries, Iran
\end{abstract}

Citation: Mousavi-Roknabadi RS, Safaei-Firouzabadi H, Mousavi-Roknabadi RS, Sharifi M, Sadegh R, Mokdad M. Covid-19 electronic registry systems in iran: a review. Int J Travel Med Glob Health. 2021;9(3):113-118. doi:10.34172/ijtmgh.2021.19.

\section{Introduction}

According to the definition provided by the World Health Organization (WHO), registry systems are a collection of documents containing similar information of unique individuals that are collected systematically and comprehensively to achieve predetermined and scientific, clinical, or political goals. ${ }^{1}$ These structured systems were designed to collect data such as demographic characteristics, clinical history, and outcomes for a specific disease or condition or a particular exposure, which can be utilized to assess the identified events in the target population..$^{2-4}$ Furthermore, these systems can be used to evaluate the quality and costeffectiveness of health services and formulate the necessary evidence for policy/decision-making purposes. ${ }^{4,5}$
Registry systems have been traditional pen-and-paper methods for field survey data collection over the past decade. ${ }^{6}$ But, paper-based medical records might be incomplete, fragmented, difficult to find or read, and they need a lot of space for archives. Moreover, they cost a lot of money to record and data preservation. Since data management in this method is very inefficient, electronic registration systems have been introduced. ${ }^{7-9}$

Since 1950s, the number of disease registration systems has significantly increased, which can be attributed to the increasing attention to the effects of chronic diseases on populations' health. Today, healthcare systems use various computer software for managerial purposes as well as storing patients' information, which increases the access to data

Copyright $(\odot 2021$ The Author(s). This is an open-access article distributed under the terms of the Creative Commons Attribution License (http:// creativecommons.org/licenses/by/4.0), which permits unrestricted use, distribution, and reproduction in any medium, provided the original work is properly cited. 
and their analyses. The ultimate goal of these systems, often supported by the government and hospitals, is public health surveillance and research. Since 2014, the Ministry of Health and Medical Education (MoHME) of Iran has launched many registry systems for various diseases and health outcomes, which till now more than 50 systems have been confirmed and received financial support. ${ }^{3}$ Meanwhile, for communicable diseases, several registries have been designed and launched in Iran. ${ }^{10-12}$

In December 2019, a novel type of coronavirus was emerged in Wuhan city, Hubei province (China). This pneumonic disease whose cause was unknown soon became a pandemic and was named COVID-19. ${ }^{13-15}$ According to the WHO statistics, the virus has spread to at least 156 countries and it is introduced as a pandemic. On January 30, the WHO declared an international emergency for public health. ${ }^{16}$ Despite all efforts, the disease spread to Iran. ${ }^{17,18}$ Due to the ambiguities around the COVID-19 and contradictory recommendations, ${ }^{19,20}$ there is a need to design and set up an electronic patient registration system. Since the beginning of this epidemic in Iran, several electronic registration systems have been generated to record the data of infected patients with COVID-19. This review aimed to investigate the articles that described the COVID-19 electronic registry systems designed and launched in Iran to get acquainted with the design aspects, the used algorithms for patients' management, recorded data, and methods of quality assurance.

\section{Methods}

In this review, electronic literature searches were conducted to identify all studies in the field of COVID-19 electronic registry systems in Iran. Hence, Medline (accessed from PubMed), Scopus, Science Direct, and Web of Science were four databases searched up till June 22, 2020, by three authors [RSM, HS, RSM]. MeSH Keywords used were ("COVID-19" OR "2019 novel coronavirus" OR "2019-nCoV” OR "severe acute respiratory syndrome coronavirus 2" OR "SARSCoV-2" OR "2019nCoV” OR "Wuhan coronavirus") AND "registry*". Google search engine and "researchgate.net" were also reviewed manually to explore the grey literature [RSM, RS]. To ensure literature saturation, the reference lists of the included studies or relevant reviews identified through the search were scanned. No time restriction was considered for the searching, and all searches were performed in English. Studies, which were not relevant to the study's aim, written in a non-English language, or studies that considered other coronaviruses except COVID-19, as well as studies without available full-text were excluded, through reading the title and the abstract [RSM, MS, RSM]. The studies were selected independently and the results were discussed to make the final selection. After reading the full text of all potentially eligible articles, a final decision was made for each study. Also, the data extraction was performed by all authors. Any disagreement was discussed after the completion of the data collection process.

The following data were extracted from the included studies: study authors, name of a registry, source of registry, type, initiation date, patients, the algorithm of patient care, recorded data, follow-up duration, and quality assurance. A data extraction form was designed in a Microsoft Excel sheet 2013, by two authors [RSM, HS]. The disagreements were resolved, and then the obtained results were summarized [RSM].

\section{Results}

After careful screening, four articles were confirmed and enrolled in this review, ${ }^{4,21-23}$ which are summarized in Table 1. These registries were designed and launched by Isfahan, Shiraz, Tehran, and Ilam Universities of Medical Sciences. They were different in design, the used algorithms for patients' management, recorded data, and methods of quality assurance. In the following, we will explain their various aspects.

Haghjooy Javanmard et $\mathrm{al}^{4}$ designed and implemented a registry system called Isfahan COVID-19 Registry (I-CORE), which as noted by them, is the first comprehensive critical incident registry system for COVID-19 in Iran. This system collects data on patients with a definitive or suspected diagnosis of COVID-19 based on the temporary guidelines developed by WHO. All patients with a definitive diagnosis of COVID-19, regardless of clinical signs and symptoms, were defined as definitive patients. Patients without a definitive COVID-19 diagnosis were categorized as a suspected case if having one of the following conditions: (1) asymptomatic patients with acute respiratory illness and a history of travel during the past 14 days to areas that local spread of the COVID-19 is reported; (2) patients with presentations of acute respiratory illness who had contact with a definitive or suspected case of COVID-19 within 14 days before the onset of the symptoms; and (3) patients with acute respiratory infection who required hospitalization and had no other etiology to justify their clinical presentations. Data were collected using WHO questionnaires and protocols. The committee included the vice-chancellor of research, health, and treatment of Isfahan University of Medical Sciences, specialized physicians, nurses of hospital infection control, and health information technology staff control the quality assurance.

They reported that by March 11, 2020, data from 3,083 patients (56\% male) with a definitive or suspected diagnosis of COVID-19 were recorded in the system. In total, 611 cases were definitive (61\% male), with a mean \pm standard deviation age of 58.44 (17.0) years (range; $14-99$ ), of which about $5.6 \%$ of them died and $32.2 \%$ were discharged from the hospital. Not describing the details of the process of collecting data and not providing an algorithm for patient care are the disadvantages of this study. Also, the duration of patients' follow-up was not mentioned in the system.

Another electronic web-based registry system in Shiraz city (south of Iran) was designed by Akbari et $\mathrm{al}^{21}$ to record data on suspected or confirmed COVID-19 patients. This system is approved by Shiraz University of Medical Sciences; it is designed based on a comprehensive form that was according to a checklist recommended by WHO. Inclusion criteria 





consisted of fever, cough, and shortness of breath. COVID-19 infection should be confirmed through chest computed tomography (CT) scan and polymerase chain reaction (PCR) test. Recorded data include patients' demographic characteristics, results of the chest CT scan, laboratory tests, complications during hospitalization, treatment, and intensive care unit (ICU) courses. Data for the hospital stay and one-month follow-up were also recorded in the system. Moreover, in this system, the virtual status of the patients' family members was monitored through phone calls, to see whether they need screening and hospitalization or not. Data on pregnant women and neonates were recorded, as well. In addition to the patient care algorithm, the authors described the flowchart of risk assessment in pregnant women as well as infants of suspected or definitively diagnosed mothers, individually.

The authors reported that due to the unknown nature of the disease, treatment protocols and guidelines may change during the study implementation. For quality assurance, a team of specialists comprised of all related fields monitored the data collection process. One of the weaknesses was not providing statistical reports about the registered patients in the manuscript published by the authors so that it seems necessary to record, analyze and report the volume of the patients' data over a period of time to determine if the system is working properly or not. Also, one of the exclusion criteria is patients' dissatisfaction to record their data in the system, while recording data in the registry systems does not require obtaining the patients' consent and all data must be recorded, so that epidemiological analyses can be performed. Similar to the I-CORE system, it was not mentioned that who collected and entered the data.

Talebpour et $\mathrm{al}^{22}$ developed a hospital-based system called Sina hospital COVID-19 Registry (SHCo-19) in Tehran, the capital of Iran. This system records data on clinical presentation, diagnostic measures, treatments, hospital courses, and the patients' follow-up who were referred to the emergency department of Sina hospital, affiliated with Tehran University of Medical Sciences, and one of the centers specially allocated to COVID-19 patients. The data were recorded retrospectively. The inclusion criteria included age $\geq 18$ years with suggestive symptoms for COVID-19 (i.e. fever, cough, and dyspnea), chest CT scan indicating the infection, and a positive PCR test. For patients who are suspected of COVID-19, the chest CT scan, laboratory tests such as complete blood cells, C-reactive protein (CRP), serum creatinine and sodium and potassium, magnesium, vitamin $\mathrm{D}$ level, liver function tests, troponin, ferritin, and checking the blood group are performed. Other diagnostic modalities are used based on the predetermined protocols. During hospitalization, patients were monitored in terms of blood pressure, fever, respiratory symptoms, oxygenation, serum electrolytes, medications, and death. Meanwhile, complications that occurred included respiratory complications (such as ARDS and secondary bacterial infections), cardiac (such as myocarditis, acute coronary syndrome, and arrhythmic), and renal complications (such as oliguria and decreased serum creatinine) monitor.
Using a checklist, data are collected separately or electronically through a web-based program by trained staff. After discharge or death of the patients, data are extracted from their medical records as well as checklists by doctors, nurses, and researchers, and enter into this web-based system. Similar to the COVID-19 registry system developed in Shiraz, ${ }^{21}$ patients and their families are monitored during the hospitalization and one month later for vital signs, readmission, and drugs side effects. In addition, as mentioned for the COVID-19 registry system of Shiraz, ${ }^{21}$ the author noted that due to the unknown nature of the disease, guidelines and protocols may change during the study. Therefore, a committee of experts continuously monitors national recommendations, data collection issues, and data entry accuracy. Retrospective data recording is one of the disadvantages of this system. However, using pre-designed checklists reduces bias and missing the data.

Finally, the authors mentioned the following advantages for this system: (1) identifying characteristics, underlying diseases, and previous risk factors of patients; (2) assessing the outcomes and better understanding of the risk factors associated with adverse events; (3) evaluating changes in the laboratory findings and their association with the poor outcomes; (4) evaluating and comparing different treatment strategies as well as their efficacy and safety; and (5) creating a platform for further clinical trial studies.

An electronic web-based registry system for COVID-19 with the support of Ilam University of Medical Sciences was designed and developed by Kazemi-Arpanahi et al. ${ }^{23}$ According to the information provided in their report, it seems that this system is more accurately designed compared to the aforementioned systems. As mentioned by the authors, initially, they were intended to record essential data and having a valid template in this registry. Then, in the second phase, the system was designed on a web-based platform. To identify essential data, first, an extensive review was performed on various medical databases, including Web of Science, Science Direct, Embase, Scopus, Cochrane, PubMed, and Google Scholar using the definite English keywords. Finally, 18 articles were selected. A checklist was then designed, whose validity was assessed by five experts using a Likert scale to determine the degree of importance of each data ('very important' to 'not very important). The test-retest was utilized to determine its reliability. The initial content was validated during two rounds of Delphi by a group of multidisciplinary specialists from hospitals affiliated with Ilam University of Medical Sciences. Weight was determined for each data and the degree of agreement for each data was calculated. Finally, after deleting 31 variables, 30 variables were selected for the non-clinical subgroup and 4 classes with 26 variables were selected for the clinical subgroup. It is noteworthy that the authors specified the type of each data (Integer, Binary, Categorical, String, and Date). The subclinical data subgroup includes the following: sociodemographic, identifier, and patients' disposition. The clinical data subgroup includes the following: diagnosis, exposure, clinical examination, medical and diagnostic procedures. 
The authors noted that the main goal of developing the platform was increasing access to the data and being userfriendliness, to speed up the reporting of COVID-19 data. Moreover, users can do a specific search on the database of the system and obtain daily statistics and multimedia instructions. Access to this system is possible for the registered members; so that each user has a unique password and username to log in. The possibility of contacting the managers of the system and providing useful news about the disease (such as prevention, self-care, and medical information) are other features of the system. Another advantage of the system is the auto-rejection of incorrect values and out-of-range data. Furthermore, it has been attempted to avoid manual data entry as much as possible. They stated that this is a comprehensive registry of COVID-19 and can provide an in-depth description of specific patient groups instead of providing epidemiological data. In this article, the authors showed some figures from a general overview of this registry, which similar articles did not provide. The weaknesses of this study include not reporting of the target group, duration of patients' follow-up, and quality assurance. In addition, the authors did not mention the algorithm of patient care. Similar to the study conducted by Akbari et $a l,{ }^{21}$ this study also did not provide any statistical reports of the registered patients.

\section{Discussion}

Studies conducted based on well-designed and wellcompleted registries provide a more realistic overview of clinical procedures, patients' outcomes, safety, efficacy, and effectiveness. Meanwhile, they support the decision-making process and evidence-based design. ${ }^{24}$ Add to this, the quality of clinical registries can be limited by the lack of unreliable data entry. On the other hand, manual data entry is timeconsuming and prone to documentation errors (such as inaccuracies and omissions). ${ }^{25,26}$ Another important feature for any registry is the ability to interact with other health information systems that can be useful to prevent duplicate data entry ${ }^{23,27}$; therefore, data coordination, uniform data definition, and uniform capture process of each item are of crucial importance. ${ }^{28,29}$

In this review, the articles that described the COVID-19 electronic registry systems designed and launched in Iran were assessed. Due to the ambiguities around the COVID-19 and contradictory recommendations, there is a need to design and set up an electronic patient registration system. On the other hand, considering the differences between various registry systems specially designed for COVID-19, including using various algorithms for patient care, differences in target groups, using different variables, it is recommended to design and develop a single web-based registry system by the MoHME to register and follow up patients with COVID-19, so that the data of these patients can be recorded nationally and uniformly in all health care centers. Registration can either be retrospective or prospective. ${ }^{30}$ Having a single uniform system, in addition to clarifying various aspects of the disease such as patients' characteristics, risk factors, laboratory findings, effective treatments, prognosis, and the trend of the

\section{Review Highlights}

\section{What Is Already Known?}

Since the beginning of the COVID-19 epidemic in Iran, several electronic registration systems are designed and launched to record the data of infected patients. In this study, we investigated the articles that described the COVID-19 electronic registry systems in Iran.

\section{What Does This Study Add?}

It was found that various registry systems were different in the used algorithms for patient care, target population, and defined variables. Hence, it is recommended to develop a single web-based registry system by the Iran Ministry of Health and Medical Education to register and follow up the patients with COVID-19.

disease, provides the possibility of conducting comprehensive and holistic epidemiological studies in the country. What is more, using a uniform system provides the ability to compare provinces and cities. Another advantage of such systems includes investigating specific groups (such as patients with cancer, cardiovascular disease, diabetes, congenital diseases, pregnant women, etc). Also, by launching a single system and linking it to other health information systems, in addition to increasing research capacity, the educational capacity will expand. Other benefits of such systems include the possibility of evaluating the short-term and long-term outcomes of patients using different variables as well as evaluating, comparing, and improving the quality of care.

\section{Conclusion}

Considering the differences between various registry systems designed for COVID-19 in Iran, it is recommended to develop a single web-based registry system by MoHME of Iran to register and follow up the patients with COVID-19.

\section{Authors' Contributions}

RSM contributed to supervision, conception, design, acquisition of data, and writing up the manuscript. HS contributed to the conception, acquisition of data, and writing the manuscript. RSM contributed to the acquisition of data and writing up the manuscript. MS contributed to the acquisition of data and writing up the manuscript. RS contributed to the acquisition of data and writing up the manuscript. MM contributed to the acquisition of data and writing up the manuscript. All authors critically reviewed the manuscript before final submission and approved it.

\section{Conflict of Interest Disclosures}

The authors declared no conflict of interest.

\section{Ethical Approval}

Not applicable.

Funding/Support

None.

\section{Acknowledgements}

The authors express their gratitude to all the healthcare providers fighting the COVID-19 around the world. 


\section{References}

1. Brooke EM. The Current and Future Use of Registers in Health Information Systems. World Health Organization (WHO); 1974.

2. Gliklich RE, Dreyer NA, Leavy MB. Registries for Evaluating Patient Outcomes: A User's Guide. Rockville, MD: Agency for Healthcare Research and Quality (AHRQ); 2014.

3. Mojarrab S, Rafei A, Akhondzadeh S, Jeddian A, Jafarpour M, Zendehdel K. Diseases and health outcomes registry systems in I.R. Iran: successful initiative to improve public health programs, quality of care, and biomedical research. Arch Iran Med. 2017;20(11):696-703.

4. Javanmard SH, Nasirian M, Ataei B, Vaseghi G, Vaezi A, Changiz T. Isfahan COvid-19 REgistry (I-CORE): Design and methodology. J Res Med Sci. 2020;25:32. doi:10.4103/jrms. JRMS_271_20.

5. Polisena J, Garritty C, Kamel C, Stevens A, Abou-Setta AM. Rapid review programs to support health care and policy decision making: a descriptive analysis of processes and methods. Syst Rev. 2015;4:26. doi:10.1186/s13643-015-0022-6.

6. Welker JA. Implementation of electronic data capture systems: barriers and solutions. Contemp Clin Trials. 2007;28(3):329336. doi:10.1016/j.cct.2007.01.001.

7. Häyrinen K, Saranto K, Nykänen P. Definition, structure, content, use and impacts of electronic health records: a review of the research literature. Int J Med Inform. 2008;77(5):291-304. doi:10.1016/j.ijmedinf.2007.09.001.

8. Brandt CA, Argraves S, Money R, Ananth G, Trocky NM, Nadkarni PM. Informatics tools to improve clinical research study implementation. Contemp Clin Trials. 2006;27(2):112122. doi:10.1016/j.cct.2005.11.013.

9. Caban-Martinez AJ, Clarke TC, Davila EP, Fleming LE, Lee DJ. Application of handheld devices to field research among underserved construction worker populations: a workplace health assessment pilot study. Environ Health. 2011;10:27. doi:10.1186/1476-069x-10-27.

10. Ershadi F, Hatam N, Askarian M, Palenik CJ. The first digital immunization registry for healthcare workers in Iran. Middle East J Fam Med. 2018;16(2):106-113. doi:10.5742/ mewfm.2018.93248.

11. Keshavarz S, Hatam N, Askarian M, Palenic CJ. Post-Exposure Procedures including Prophylaxis : First National Electronic Registry for Iran. Middle East Journal of Family Medicine. 2018;16(2):114-22. doi:10.5742/mewfm.2018.93249.

12. Sarbizhan HR, Hatam N, Askarian M. The first electronic immunization registry system in Iran, Iranian Immunization Registry (IIR). Middle East J Fam Med. 2017;15(6):101-110. doi:10.5742/mewfm.2017.92994.

13. Şahin AR, Erdoğan A, Ağaoğlu PM, et al. 2019 novel coronavirus (COVID-19) outbreak: a review of the current literature. Eurasian J Med Oncol. 2020;4(1):1-7. doi:10.14744/ ejmo.2020.12220.

14. Olagunju AS, Oladapo IP, Kosemani SO, Olorunfemi FG, Adeyemo AS. Pathogenesis of severe acute respiratory syndrome coronavirus-2 (SARS-CoV-2) respiratory infection. Int J Travel Med Glob Health. 2020;8(4):137-145. doi:10.34172/ ijtmgh.2020.24.

15. Mohammadi SM, Ashtari S, Khosh Fetrat M. The psychological impact of COVID-19 pandemic on mental health of Iranian population. Int J Travel Med Glob Health. 2020;9(1):19-24. doi:10.34172/ijtmgh.2021.04.

16. Yuan M, Yin W, Tao Z, Tan W, Hu Y. Association of radiologic findings with mortality of patients infected with 2019 novel coronavirus in Wuhan, China. PLoS One. 2020;15(3):e0230548. doi:10.1371/journal.pone.0230548.

17. World Health Organization (WHO). WHO Coronavirus Disease (COVID-19) Dashboard. WHO; 2021.

18. Daneshpazhooh M, Mahmoudi H. COVID-19: the experience from Iran. Clin Dermatol. 2021;39(1):23-32. doi:10.1016/j. clindermatol.2020.12.009.

19. Mousavi-Roknabadi RS, Haddad F, Fazlzadeh A, Kheirabadi D, Dehghan H, Rezaeisadrabadi M. Investigation of plasma exchange and hemoperfusion effects and complications for the treatment of patients with severe COVID-19 (SARSCoV-2) disease: A systematic scoping review. J Med Virol. 2021;93(10):5742-5755. doi:10.1002/jmv.27182.

20. Kheirabadi D, Haddad F, Mousavi-Roknabadi RS, et al. A complementary critical appraisal on systematic reviews regarding the most efficient therapeutic strategies for the current COVID-19 (SARS-CoV-2) pandemic. J Med Virol. 2021;93(5):2705-2721. doi:10.1002/jmv.26811.

21. Akbari A, Moghadami M, Ghasemi Y, et al. Designing and launching coronavirus disease 19 electronic registry in Shiraz, Iran: a brief report. Int J Basic Sci Med. 2020;5(2):33-8. doi:10.34172/ijbsm.2020.02.

22. Talebpour M, Hadadi A, Oraii A, Ashraf H. Rationale and design of a registry in a referral and educational medical center in Tehran, Iran: Sina hospital COVID-19 registry (SHCo-19R). Front Emerg Med. 2020;4(2 Suppl):e53.

23. Kazemi-Arpanahi H, Moulaei K, Shanbehzadeh M. Design and development of a web-based registry for coronavirus (COVID-19) disease. Med J Islam Repub Iran. 2020;34:68. doi:10.34171/mjiri.34.68.

24. Korngut L, Genge A, Johnston M, et al. Establishing a Canadian registry of patients with amyotrophic lateral sclerosis. Can J Neurol Sci. 2013;40(1):29-35. doi:10.1017/s0317167100012919.

25. Yang M, Loeb DF, Sprowell AJ, Trinkley KE. Design and implementation of a depression registry for primary care. Am J Med Qual. 2019;34(1):59-66. doi:10.1177/1062860618787056.

26. Williams A, Goedicke W, Tissera KA, Mankarious LA. Leveraging existing tools in electronic health record systems to automate clinical registry compilation. Otolaryngol Head Neck Surg. 2020;162(3):408-409. doi:10.1177/0194599820901713.

27. Niazkhani Z, Cheshmekaboodi M, Pirnejad H, et al. Enabling informed policymaking for chronic kidney disease with a registry: initiatory steps in Iran and the path forward. Health Policy Technol. 2018;7(1):73-80. doi:10.1016/j.hlpt.2018.01.004.

28. Plotnicki L, Kohl CD, Höcker B, et al. The CERTAIN registry: a novel, web-based registry and research platform for pediatric renal transplantation in Europe. Transplant Proc. 2013;45(4):1414-1417. doi:10.1016/j.transproceed.2013.01.007.

29. Gerke AK, Tang F, Cozier YC, et al. A web-based registry for patients with sarcoidosis. Sarcoidosis Vasc Diffuse Lung Dis. 2017;34(1):26-34. doi:10.36141/svdld.v34i1.5129.

30. Harriman SL, Patel J. When are clinical trials registered? an analysis of prospective versus retrospective registration. Trials. 2016;17(1):187. doi:10.1186/s13063-016-1310-8. 